\title{
Metrology of the LHC Dipole Cold Masses
}

\author{
M. Bajkó, A. Pardons, and F. Savary
}

\begin{abstract}
In order to provide the largest possible mechanical aperture for the LHC beam, the dipole cold masses have to match the circular trajectory of the particle beam. The requirements on the dipole cold mass geometry are dictated by the beam optics of the LHC machine and by the mechanical deformation limits of the interconnection zone. The geometry of the approximately 15 $\mathrm{m}$ long, $0.57 \mathrm{~m}$ diameter, and $30 \mathrm{t}$ weight dipole cold mass is verified by the measurement of the axes of the cold bore tubes. The tight tolerances imposed, necessitate the use of a high accuracy 3-D measuring system based on optical methods. During the last 2 years, 6 prototypes and 4 pre-series magnets have been assembled at CERN. The summary of the results obtained on these cold masses is presented, as well as the evolution of the tooling and the measuring method.
\end{abstract}

Index Terms-3-D measurement, alignment, LHC dipole cold mass assembly.

\section{INTRODUCTION}

$\mathbf{T}$ HE SERIAL production of the dipole cold masses has started. During the period of the prototype and pre-series fabrication, the assembly took place partly at the magnet manufacturer's premises and partly at CERN: the collared coils were prepared at the magnet manufacturer's premises, while cold masses (CM) were assembled at CERN, in collaboration with the manufacturer's personnel. The collared coil is a laminated structure containing the two magnetic elements of the dipole cold mass, i.e. the superconducting coils. This paper presents the experience gained on the CM assembly at CERN, during prototype and pre-series assembly.

As the LHC 15-m long dipoles are curved magnets, one of the most critical assembly operations is the shaping of the CM. Therefore, a special effort was made to determine the best combination of the influencing parameters, i.e., the initial shape of the half cylinders and the form of the welding press table [1].

Tolerances on the shape and on the position of some of the $\mathrm{CM}$ components are of order of magnitude of tenths of millimeters. Therefore, in-situ 3-D dimensional measurements are performed during several assembly steps.

\section{The Tolerances on the Dipole Cold Mass Geometry}

The reference plane of the dipole cold mass is the plane containing the two theoretical axes of the two cold bore tubes (CBT) called V1 and V2. These axes consist of a curved part in the center and two tangent lines in the ends. The transversal sagitta over the magnetic length of $14.3 \mathrm{~m}$ is $9.14 \mathrm{~mm}$, given by the bending radius of $2803.92 \mathrm{~m}$ at $1.9 \mathrm{~K}$. The distance between the two CBT axes is $194.52 \mathrm{~mm}$ at nominal working conditions.

Manuscript received September 24, 2001.

The authors are with CERN, Geneva, CH (e-mail \{Marta.Bajko; Ans.Pardons; Frederic.Savary\}@ cern.ch).

Publisher Item Identifier S 1051-8223(02)04273-2.

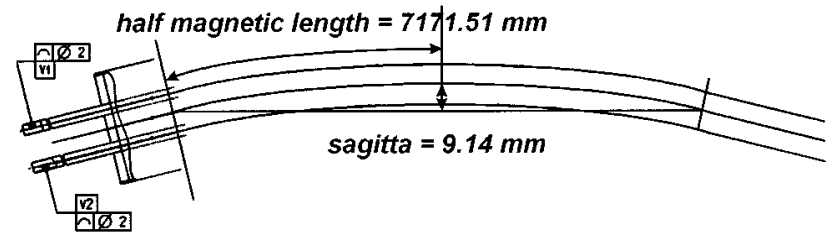

Fig. 1. The shape tolerance of the dipole cold mass.

All tolerances of the assembly are with respect to the references plane or the axes V1 and V2.

\section{A. The Shape Tolerance}

Along the arc, two toroidal sectors are generated by a circle of $1 \mathrm{~mm}$ radius, moved along the circumference of the theoretical geometric axes of each beam channel. In the straight ends, a $1 \mathrm{~mm}$ radius cylinder represents the tolerance range. The combination of the toroidal sectors with the straight cylinders gives the shape tolerance for the axis of each aperture (see Fig. 1).

\section{B. Tolerance on the Corrector Magnet Positioning}

The dipole magnets are equipped with corrector magnets. Type "B" dipoles have a sextupole corrector magnet at one extremity while type "A" magnets have an additional decapole/octopole corrector magnet at the other extremity. Since corrector magnets correct the chromaticity and the persistent current effects of the superconducting cables, they should be positioned on the beam trajectory. The tolerances of these magnets on the ends of the dipoles is represented by a $0.3 \mathrm{~mm}$ radius circle centered on V1 or V2.

\section{The Tolerances on the End Cover Positioning}

The end covers of the dipole CM are important for the interconnections of the dipoles. They carry the necessary passages for a number of elements to be connected together in the machine. There are bellows as interconnecting elements, but the longitudinal elongation between the room temperature and nominal working temperature of $1.9 \mathrm{~K}$ limits their transversal flexibility to $4 \mathrm{~mm}$. In order to assure the best conditions for the interconnections in the tunnel, the positioning tolerances on the end covers at the assembly stage are the following:

1) The perpendicularity of the end covers with respect to the reference plane should be better than $0.2 \mathrm{~mm}$, i.e., an angle between cover and plane of less than 0.36 mrad.

2) The vertical symmetry plane of the end covers has to be positioned with respect to the central geometrical axes to within $1 \mathrm{~mm}$.

3) The horizontal symmetry plane of the end cover has to be positioned with respect to the reference plane V1V2 to within $1 \mathrm{~mm}$. 


\section{Tolerances of the Cold Bore Tubes Position}

Since the interconnections of the beam channels are realized with flexible bellows, the tolerance on the tube ends reflects the limit of vertical and transversal deflection of the interconnects. To fall within the tolerance range, the position of the center of the CBT extremity should stay within a radius of $0.3 \mathrm{~mm}$ around the reference axes $\mathrm{V} 1$ or $\mathrm{V} 2$.

\section{E. Tolerance on the Cold Feet Pads}

The cold feet pads are the elements connecting the CM to the cryostat feet. They should be positioned $5400 \mathrm{~mm}$ distant within $\pm 1 \mathrm{~mm}$ and the plane of the feet should be parallel with the reference plane to within $0.5 \mathrm{~mm}$, meaning that the angle between plane and feet should not exceed $1.67 \mathrm{mrad}$ around the transversal and $1.38 \mathrm{mrad}$ around the longitudinal axis [1].

\section{CURVING THE Magnets}

In order to weld the half cylinders, the CM is rotated $90 \mathrm{deg}$ with respect to its normal working position, i.e., the inner half cylinder becomes the lower and the outer half cylinder becomes the upper. In this position the shaping operation is as follows:

1) The table of the welding press is shimmed to follow a curvature equal or similar to that of the nominal shape of the dipole CM.

2) The assembly of the collared coils with the magnetic laminations is put between the two half cylinders and is placed on the table prepared as in 1 .

3) Due to its self-weight, the assembly takes the shape of the table. The press load is applied on the magnet.

4) The two half cylinders are welded longitudinally. When the load is released, the magnet loses a nonnegligible fraction of the curvature due to elastic spring back.

The initial curvature of the half shells and the shape of the welding-press table influence the spring back. As the CM assembly is a nonhomogeneous structure, it is difficult to predict the optimum parameters for the shaping. Several trials with different values for these parameters were made, resulting in some cold masses being insufficiently or too strongly curved.

\section{A. The Half Cylinders}

The half cylinders are made of an austenitic stainless steel. They are cylindrical half tubes of a length of $15.35 \mathrm{~m}$ an inner diameter of $0.55 \mathrm{~m}$ and a thickness of $10 \mathrm{~mm}$. They weigh approximately $1.1 \mathrm{t}$. During the construction of the prototype magnets, straight half cylinders were used, consisting of three welded parts of $6 \mathrm{~m}$ length. For the pre-series production, the two shells had the same sagitta of $9 \mathrm{~mm}$, one shell being concave and the other convex. In order to achieve an optimum combination of the shape of the welding press table and the form of the shells, an additional case was studied: half shells with both inner and outer cylinders bent in the same direction but with a more accentuated sagitta, approximately $12 \mathrm{~mm}$. In those cases the table of the welding press was adapted proportionally.

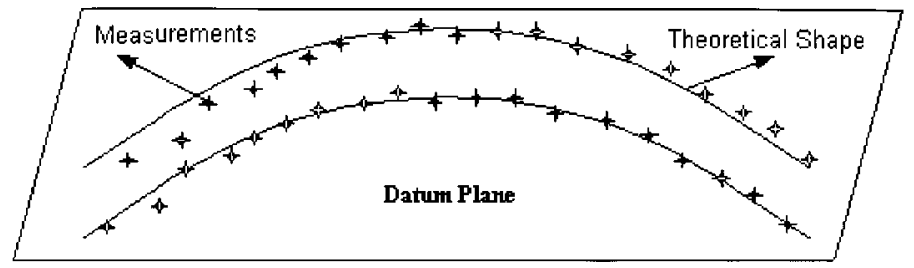

Fig. 2. Datum plane determination by best fitting the measurements with the theoretical shape.

\section{3-D DIMENSIONAL INSPECTION}

The shape of the dipole cold mass is represented by the shape of the CBT axes. To determine the datum plane for the next steps of the assembly, the CBT axes should be measured and compared with the theoretical axes of the cold mass. The tubes have a $50 \mathrm{~mm}$ diameter and are about $16 \mathrm{~m}$ long. To measure the axes, the sole valid measuring techniques that assure the required accuracy at the given conditions rely on optical methods. The measuring instrument used is a LTD500 laser tracker based on interferometric techniques. A mechanical mole centered with respect to the CBT holds a reflector as target while traveling through the tube. The position of this reflector, describing the axes of the CBT, is measured with the LTD500 in a common Cartesian coordinate system. In order to determine, with high accuracy, the reference plane of the cold mass, the axes of the cold bore tubes must be measured from both sides of the CM. This is inherent to the fact that the precision of the 3-D optical measuring system degrades proportionally with the distance from the laser head to the measured point.

In order to get comparable data for the calculations, the theoretical axes of the CBT are discretized in a number of points equivalent to the number of measurements taken. The measurements matrix, built with the 3-D coordinates of the centers of the two cold bore tubes, is best fitted with the theoretical geometry expressed in a similar matrix by the most general method of transforming coordinates, using seven geometrical transformation parameters. In this way, any translation of the origin, rotation of the axes and scale change may be accommodated. The general formulation of this transformation is usually attributed to the German geodesist: Helmert. The transformation parameters are three translations three rotationangles and a scaling factor.

After the transformation, all measurements can be expressed in the datum plane's coordinate system (1).

$$
\begin{array}{r}
\left|\begin{array}{l}
x m_{1_{j}} \\
y m_{1_{j}} \\
z m_{1_{j}} \\
x m_{2_{k}} \\
y m_{2_{k}} \\
z m_{2_{k}}
\end{array}\right|=\left|\begin{array}{c}
t x \\
t y \\
t z
\end{array}\right|+\left(1+\delta_{m}\right)\left|\begin{array}{ccc}
1 & \gamma & -\beta \\
-\gamma & 1 & \alpha \\
\beta & -\alpha & 1
\end{array}\right|\left|\begin{array}{l}
x t_{1_{j}} \\
y t_{1_{j}} \\
z t_{1_{j}} \\
x t_{2_{k}} \\
y t_{2_{k}} \\
z t_{2_{k}}
\end{array}\right| \\
(j=0,1, \ldots n) \quad(k=0,1, \ldots m) .
\end{array}
$$

Where the indices 1 and 2 denote the two cold bore tubes and $n$ and $m$ are the number of measurements made on each of the cold bore tubes. $T x, t y, t z$ are the translation parameters, $\alpha, \beta, \gamma$ the rotation parameters and $\delta m$ the scaling factor. 


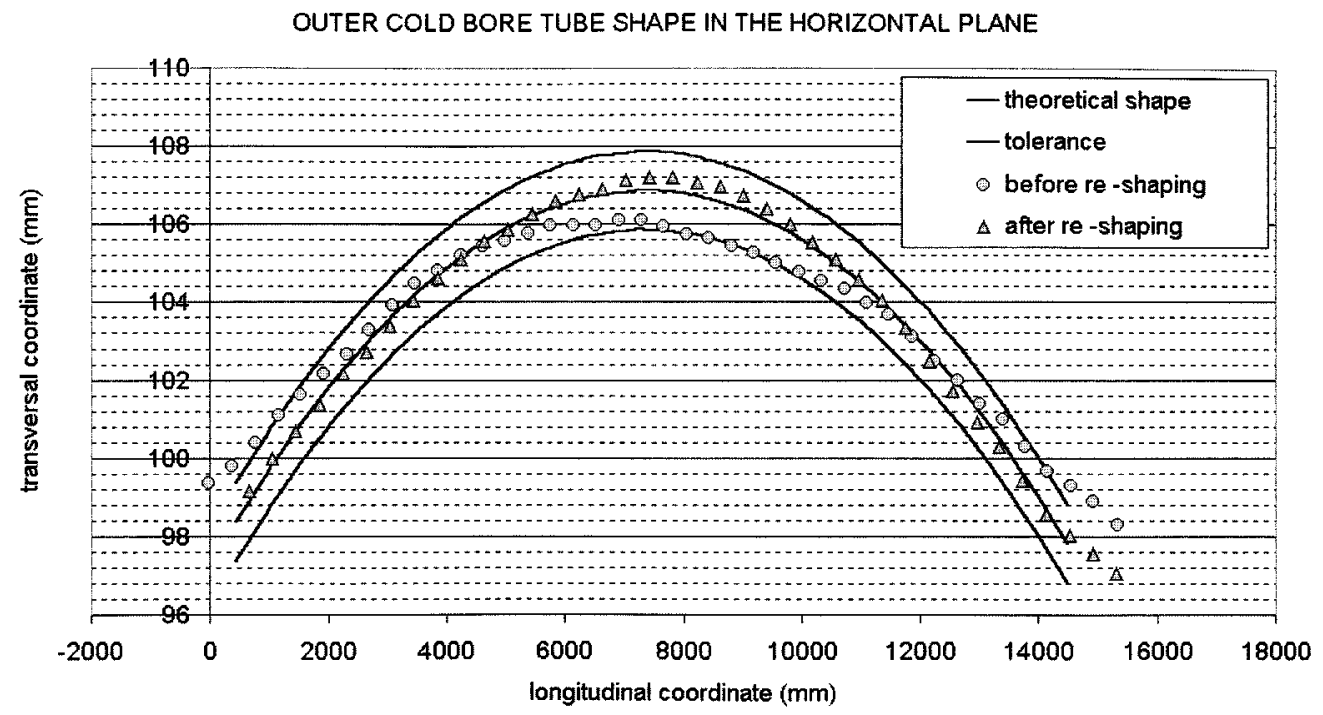

Fig. 3. CBT axis before and after repairing the horizontal shape of a CM.

TABLE I

CURVATURe PARAMETERS AND RESUlting CM SHAPE

\begin{tabular}{lllllll}
\hline Parameter & \multicolumn{7}{c}{ Value } \\
& \multicolumn{7}{c}{15} \\
Table sagitta [mm] & 15 & 9 & 9 & 15 & 12 & 11 \\
Shell sagitta [mm] & 0 & 9 & 12 & 12 & 12 & 12 \\
CM sagitta [mm] & 9 & $<9$ & $<9$ & $>9$ & $>9$ & 9 \\
\hline
\end{tabular}

An external network system is measured together with the CBT axes and expressed in the datum plane coordinate system. Measuring this network system and making the same 3-D data transformation on the values expressed in the datum plane system allows the positioning and the checking of the different components of the assembly. The only requirement is the stability of the network with respect to the CM and vice versa. With this condition fulfilled, the components can be positioned with an accuracy that is only limited by the precision of the positioning tools.

A semi-automated measuring system is created to guide the operators during the measurements and positioning operations, as well as to reduce time and the possibility of errors using a sophisticated measuring system during the construction of the dipole cold masses.

\section{RESULTS}

\section{A. The Curvature}

After some iteration, a situation where the press table is shimmed to a sagitta of $11 \mathrm{~mm}$ and where both half shells are concave with a sagitta of $12 \mathrm{~mm}$ was retained as an optimum solution. The different trials and their qualitative results are listed in Table I.

In the case of unacceptable sagitta after longitudinal welding, a corrective action is possible by locally deforming the CM into the plastic range. The operation needs a rigid structure in order to apply the necessary loads. Forces up to $12 \mathrm{t}$ were applied on the extremities of the CM and absolute deflections up to $15 \mathrm{~mm}$
TABLE II

POSITION OF THE END COVERS ON DIFFERENT COLD MASSES [MM]

\begin{tabular}{|lcccccc|}
\hline side of the CM & axis & \multicolumn{5}{c|}{ CM } \\
\hline & & serie 1 & seri2 & serie 2 & seri3 & serie 3 \\
\hline upstream & \multirow{2}{*}{0.22} & 0.13 & 0.1 & 0.13 & -0.16 \\
downstream & $\mathrm{y}$ & 0.27 & $\mathbf{- 1 . 0 3}$ & 0.06 & -0.08 & 0.14 \\
upstream & & -0.001 & 0.35 & 0.37 & -0.02 & -0.26 \\
downstream & $\mathrm{z}$ & 0.077 & $\mathbf{0 . 6 3}$ & 0.16 & -0.2 & 0.4 \\
\hline
\end{tabular}

were measured to obtain the needed $1-1.2 \mathrm{~mm}$ residual deformation. From a 10 magnets assembled at the Magnet Assembly Facility (MAF) at CERN, 9 magnets were within the tolerance after assembly, of which 6 were reshaped after the longitudinal welding. Fig. 3 shows the shape of the outer CBT axis before and after re-shaping a $\mathrm{CM}$. This $\mathrm{CM}$ was repaired because of an insufficient sagitta in the horizontal plane after the longitudinal welding.

\section{B. The Corrector Magnets}

In the case of the first two prototype cold masses, the location of the corrector magnets was measured but, since no alignment tool existed, it was not possible to adjust their position. An appropriate aligning tool was designed and Fig. 4 shows that the corrector magnets of all subsequent cold masses were within the tolerances.

\section{The End Covers}

As the end covers are welded on the two ends on the shrinking cylinders, their position is guided by the extremity of the cylinders. Difficulties during positioning of the end covers typically arise from a shape error. An over-bent or insufficiently bent cold mass cylinder end is still acceptable with up to $1 \mathrm{~mm}$ eccentricity with respect to the main axis of the CM. The tolerance on the end cover location is only $0.5 \mathrm{~mm}$ and the only way to compensate the remaining $0.5 \mathrm{~mm}$ is to a make the welding joint between the shrinking cylinder and end cover slightly eccentric. To assure the good quality of the welding, this eccentricity should not exceed $0.7 \mathrm{~mm}$. The results obtained on the positioning of 


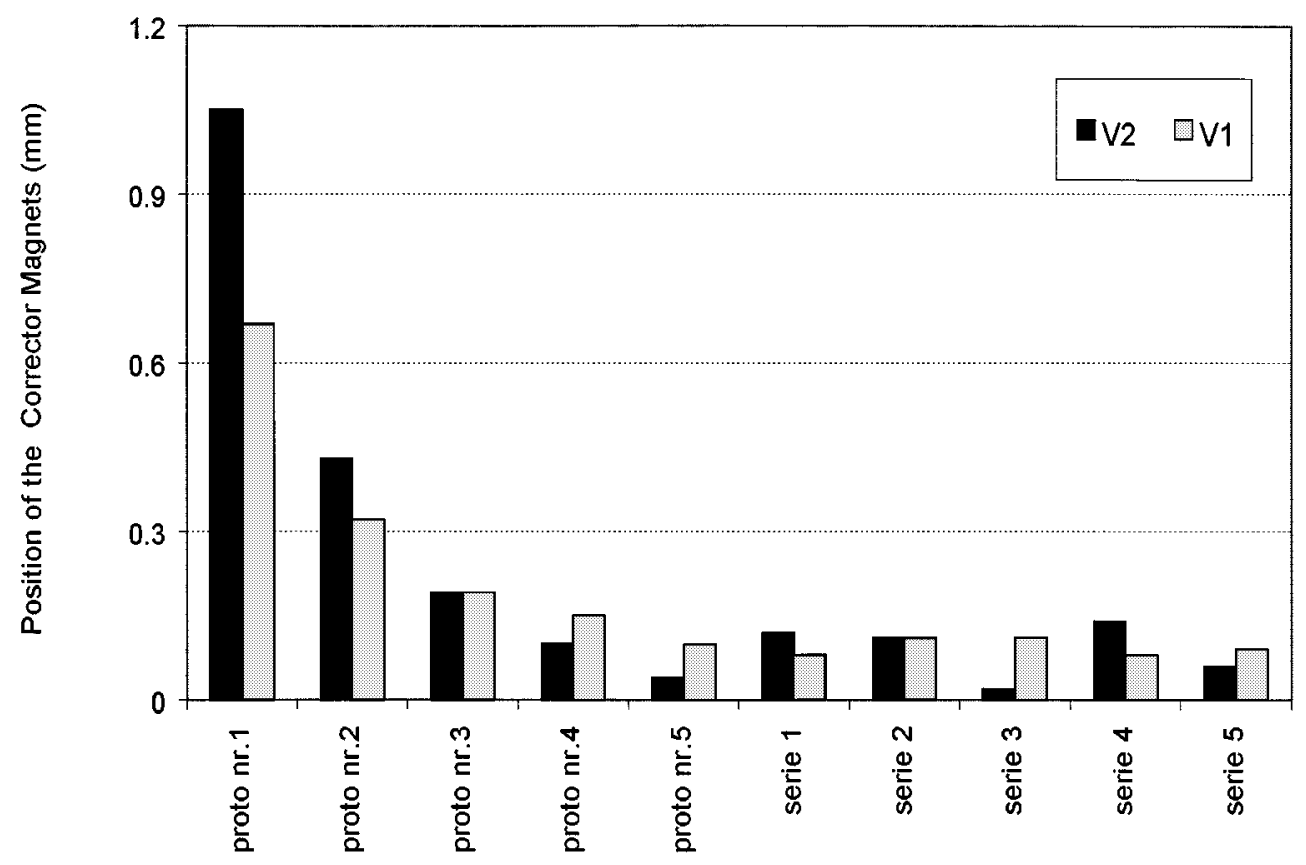

Fig. 4. Position of the corrector magnets in the different cold masses.

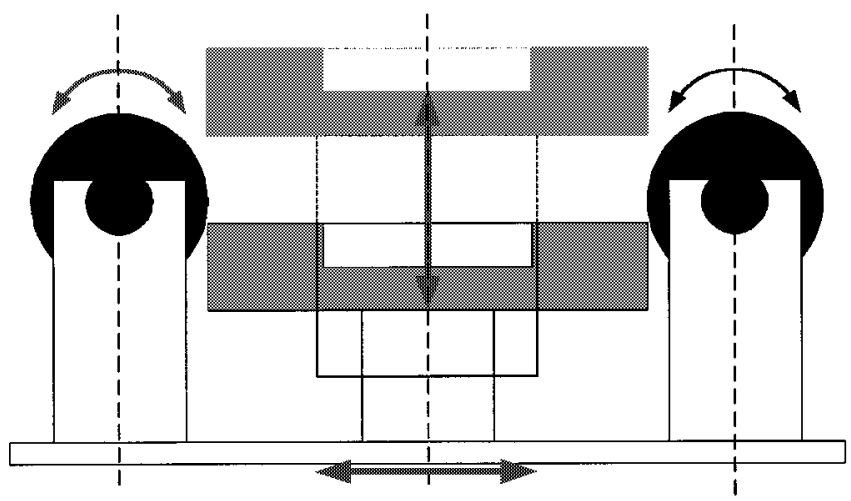

a.



b.

Fig. 5. The cold feet pads positioning tool. (a) The central pad support, (b) the external pads support.

the end covers on the pre-series cold masses of the final design are shown in Table II. Note that the nominal position is zero for both sides and both axes.

The angular position of the end covers around the longitudinal and vertical axes of the CM depends on the preparation of the end of the shrinking cylinder and on the sequence of the tack welding, which fixes the cover to the cylinder before welding. The measured values of these angles on the different cold masses vary between 0 and 1.1 mrad.

\section{The Cold Feet Pads}

The cold feet pads (CFP) are the interfaces between the CM and the cryostat. They are fixed on the CM by welding. A special tool was developed at CERN for positioning the pads on the CM. Since the CM is supported on three feet, the tooling for the CFP consists of 3 main parts: one for the central pad and one for each extremity pad. All three parts can be individually adjusted in the longitudinal direction. The two external supports can be rotated around its vertical axes, while the central support is free for horizontal movements. In this way the supports will never constrain the dipole CM. Therefore, during the whole assembly, the CM will be only deflected under its self-weight.

The tool can hold the pads in a low or in a high position. The low position is used during the adjusting of the feet in the longitudinal direction. The high position takes over once the feet are positioned and ready for the welding operation. The tool will remain at the high position during the further assembly. The degrees of freedom of each of the supports are shown in Fig. 5.

The angular misalignment of the cold feet pads is the most critical point for the interconnection between the cryostat and CM. As the pads are posed on the tool during the positioning and the welding to the CM, their angular positioning is entirely defined by the tool. Therefore, the position of this tool should be 


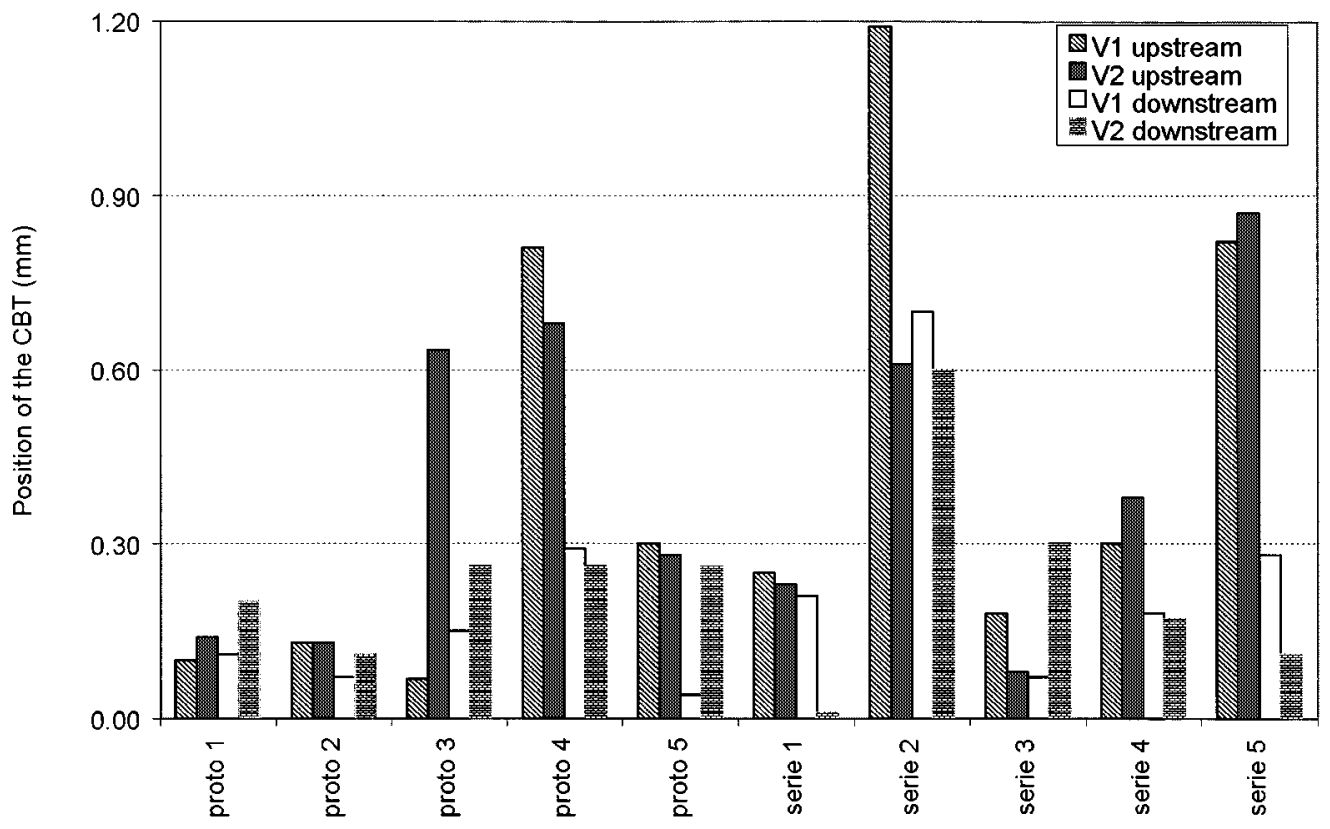

Fig. 6. Position of the cold bore tube ends on the different cold masses.

verified periodically, e.g., after $10 \mathrm{CM}$ assemblies. The angles measured on the different cold masses on the different feet and around both transversal and longitudinal axes are in the range $0.27-2.62 \mathrm{mrad}$. Three nonconformities on the angular positioning were found: the tolerance on the central foot was exceeded twice by $1 \mathrm{mrad}$ and one of the external foot tolerances was exceeded once by $0.3 \mathrm{mrad}$.

\section{E. The $C B T$}

Welding the cold bore tube extremities to the end covers is one of the last positioning operations. A $1 \mathrm{~mm}$ clearance between CBT and collared coils permits the tubes to be placed in their nominal position. In a next step, end flanges are welded to the extremity of each tube. These end flanges should, after welding, still be fixed as precisely as the cold bore tubes were positioned. Fig. 6 shows the measurement results on the different cold masses. For the first 6 dipoles, the position of the ends is measured before welding the flange. Starting from the magnet "serie2," we measured on the flanges, which means that the error induced during welding is included in the result. The technique of the positioning and welding of those flanges on the ends of the tubes is not yet fully defined and we are still looking for a tool to help this operation.

\section{CONCLUSIONS}

After a few iterations at the Magnets Assembly Facility at CERN, with different configurations of the shape of the welding press table and of the half cylinders, a combination of those parameters giving a good result was found. Cold masses with unacceptable sagitta after the longitudinal welding were corrected successfully by plastic deformation. The final assembly steps assisted by 3-D dimensional inspection were determined. The prescribed tolerances of the final shape of the cold masses and of their various components were found attainable. The most critical ones are those required on the position of the CBT extremity flanges. They are under revision.

\section{ACKNOWLEDGMENT}

The authors thank the team of the Magnet Assembly Facility at CERN for their help to carry out the 3-D measurements assisted assembly of 10 dipole cold masses for the LHC.

\section{REFERENCES}

[1] M. Bajko, F. Savary, and W. Scandale, "Geometry and alignment requirement for the LHC main dipole," in EPAC 2000, Vienna, Austria, 2000. 